\title{
Evaluation of prognostic markers for patients with curatively resected thoracic esophageal squamous cell carcinomas
}

\author{
MASAHIDE IKEGUCHI ${ }^{1}$, YUSUKE KOUNO ${ }^{1}$, KYOICHI KIHARA ${ }^{1}$, \\ KAZUNORI SUZUKI ${ }^{1}$, KANENORI ENDO ${ }^{1}$, SEIICHI NAKAMURA ${ }^{1}$, TAKASHI SAWADA ${ }^{1}$, \\ TETSU SHIMIZU $^{1}$, TOMOYUKI MATSUNAGA ${ }^{2}$, YOJI FUKUMOTO ${ }^{2}$ and HIROAKI SAITO ${ }^{2}$ \\ ${ }^{1}$ Department of Surgery, Tottori Prefectural Central Hospital, Tottori $680-0901 ;{ }^{2}$ Department of Surgery, \\ Division of Surgical Oncology, Faculty of Medicine, Tottori University, Yonago 683-8504, Japan
}

Received January 3, 2016; Accepted October 17, 2016

DOI: $10.3892 /$ mco.2016.1073

\begin{abstract}
The Glasgow Prognostic Score (GPS), neutrophil/lymphocyte ratio (NLR) and prognostic nutritional index (PNI) are prognostic parameters for malignancies. Additionally, serum squamous cell carcinoma antigen (SCC-Ag) and cytokeratin 19 fragments (CYFRA 21-1) are tumor markers for squamous cell carcinoma. In the present study, the prognostic importance of these markers in patients with resectable thoracic esophageal cancer was investigated. In this retrospective study, 84 enrolled patients diagnosed with resectable clinical stage I-III thoracic esophageal squamous cell carcinomas (ESCCs) underwent thoracic esophageal resection and three-field lymph node dissection at Tottori University Hospital between January 2007 and December 2013. The correlations among preoperative patient markers (GPS, NLR, PNI, SCC-Ag and CYFRA 21-1) and the occurrence of postoperative complications and patient survival were analyzed. The operative mortality was $2.4 \%$, and morbidity was $42.9 \%$. Strong correlations between occurrence of postoperative complications and open thoracotomy $(\mathrm{P}=0.083)$ and high-serum CYFRA 21-1 $(\mathrm{P}=0.007)$ were observed. In 15 patients with high-serum CYFRA 21-1, postoperative complications were detected in
\end{abstract}

Correspondence to: Dr Masahide Ikeguchi, Department of Surgery, Tottori Prefectural Central Hospital, 730 Ezu, Tottori 680-0901, Japan

E-mail: ikeguchim@pref.tottori.jp

Abbreviations: ALB, albumin; CRP, C-reactive protein; CT, computed tomography; CYFRA 21-1, cytokeratin 19 fragments; DFS, disease-free survival; ECOG-PS, Eastern Cooperative Oncology Group Performance Status; ESCC, esophageal squamous cell carcinoma; GPS, Glasgow Prognostic Score; NAC, neoadjuvant chemotherapy; NLR, neutrophil/lymphocyte ratio; OS, overall survival; PNI, prognostic nutritional index; SCC-Ag, squamous cell carcinoma antigen

Key words: neutrophil/lymphocyte ratio, esophageal squamous cell carcinoma, cytokeratin 19 fragments, postoperative complication, survival
11 of them (73.3\%); on the other hand, complications occurred in 25 of $69(36.2 \%)$ with low-serum CYFRA 21-1. The 5-year disease-free survival rate and 5-year overall survival rate of all the patients were 52.2 and $50.8 \%$, respectively. Among the prognostic parameters, preoperative high NLR was determined to be a poor prognostic factor, independent of the tumor stage in the multivariate analysis. These results may indicate that, in patients with preoperative high-serum CYFRA 21-1, more attention should be paid to the occurrence of postoperative complications. Therefore, in such cases, anastomosis between blood vessels of the substitute esophagus and cervical vessels would be recommended. Furthermore, in patients with high preoperative NLR, effective adjuvant chemoradiotherapy should be considered to prolong the patients' survival, even of stage I or II patients.

\section{Introduction}

The prognosis of patients with squamous cell carcinomas that occur in the thoracic esophagus remains poor. Even following curative resection, numerous patients die due to tumor recurrence. Lymph nodes metastases to the neck, chest, and abdomen have been detected frequently in resected specimens from patients with thoracic esophageal squamous cell carcinoma (ESCC) (1). Thus, subtotal esophagectomy plus three-field lymph node dissection (cervical, thoracic and abdominal) has been introduced as an effective therapeutic strategy for the treatment of localized thoracic ESCC in Japan $(2,3)$. However, due to high morbidity and mortality rates following thoracotomy and laparotomy for patients with thoracic ESCCs, the use of surgery to treat this condition has been limited $(4,5)$. A number of institutes have excluded patients with $\mathrm{T} 4$ cancer (invasion to the adjacent organs), with distant metastasis (to the lung, liver, bone, or extended lymph nodes), with poor Eastern Cooperative Oncology Group Performance Status (ECOG-PS), with severe organ dysfunction, including the cardiopulmonary system, liver, and kidney, or with an advanced age ( $>75$ years old) from surgical treatments $(5,6)$. Therefore, to set in place the best treatment program for patients with operable thoracic ESCC, it is very important to evaluate factors that indicate the occurrence of postoperative complications and prognosis of patients with thoracic ESCC preoperatively. 
With the exception of a subjective evaluation of ECOG-PS, patients have been evaluated objectively through the Glasgow Prognostic Score (GPS), calculated by the combination of C-reactive protein (CRP) and albumin (ALB), the neutrophil/lymphocyte ratio (NLR) and the prognostic nutritional index (PNI), which have been reported to be important prognostic markers in cancer patients (7-15). These markers represent the patients' chronic systemic inflammation, immune status and nutritional condition. Furthermore, serum squamous cell carcinoma antigen (SCC-Ag) and cytokeratin 19 fragments (CYFRA 21-1) have been reported as good markers of ESCC progression (16-18).

Thus, in the present study, the objective preoperative markers, GPS, NLR, PNI, serum SCC-Ag and CYFRA 21-1, were retrospectively analyzed in patients with operable thoracic ESCCs, and an evaluation was made as to whether these factors are good markers of complications and prognosis in these patients.

\section{Materials and methods}

Patients. A total of 84 patients with clinical stage I-III thoracic ESCCs underwent thoracic esophagectomy plus three-field lymph node dissection (cervical, thoracic and abdominal) at Tottori University Hospital during January 2007 and December 2013. The clinical tumor stages were routinely diagnosed by esophagogastroscopy, computed tomography (CT) scanning, an endoscopic ultrasound and positron emission topography. Clinical examination, pulmonary function tests, electrocardiography, echocardiography and exercise tolerance testing were used to assess the validity of the surgical procedures for the patients. These 84 patients with potentially resectable thoracic ESCCs were deemed to be physiologically fit for surgery.

The average age of the patients was 65.7 years old (range: 49-78 years). A total of 73 patients were males, and 11 were females. Informed consent was obtained from all the patients prior to treatment. All investigations reported in the present study were conducted in conformity with the recommendations of the Declaration of Helsinki. All patients were followed up at Tottori University Hospital until September 2015. The median follow-up period was 35.5 months (range, 1-102 months).

Operation. Traditional right antero-lateral thoracotomy with laparotomy (open method) was routinely performed in our institute at Tottori University Hospital between January 2007 and August 2009. Thoracoscopic thoracic esophagectomy in the prone position was introduced in September 2009 (the thoracoscopic method). However, the use of right anterolateral thoracotomy was indicated for those cases with strong adhesion between the lungs and the pleura. The open method was performed on 33 patients, and the thoracoscopic method was performed on 51. To diagnose the safety of resected proximal margin of the esophagus, intraoperative histological analysis of the frozen section of the proximal margin was routinely performed. The stomach was used as the substitute esophagus in 74 patients, but in 10 patients with double cancers (esophageal and gastric cancer) or for gastrectomized cases, the jejunum was elongated to cervical esophagus or pharynx via an ante-sternum root.

Chemotherapy. S-1 adjuvant chemotherapy has been introduced at Tottori University Hospital for patients with stage II or III ESCCs. According to the results from the Japan Clinical Oncology Group (JCOG) studies 9204 and 9907 (19,20), since 2010, neoadjuvant chemotherapy (NAC) with cisplatin plus 5-fluorouracil (5-FU) has been offered to patients who have been diagnosed as clinical stages II and III in our institute. Therefore, chemotherapy was performed for 45 patients (adjuvant, 7; NAC, 27; and both, 11).

Blood samples. Blood samples were obtained from each patient routinely prior to chemotherapy or surgery. Serum levels of ALB, CRP, the total lymphocyte count, serum SCC-Ag and CYFRA 21-1 were recorded. Patients with normal CRP $(<1.0 \mathrm{mg} / \mathrm{dl})$ and ALB $(\geq 3.5 \mathrm{~g} / \mathrm{dl})$ levels had a GPS of 0 , and were classified as the low GPS group. Patients with either one abnormal factor (GPS=1), or both abnormal factors $(\mathrm{GPS}=2)$ were classified as the high GPS group. An NLR $\geq 3$ was considered as abnormal (21). The PNI [PNI $=10 \times$ serum ALB concentration $(\mathrm{g} / \mathrm{dl})+0.005 \mathrm{x}$ lymphocyte count (number $/ \mathrm{mm}^{3}$ ) in peripheral blood] cut-off value was determined to be 50 (22). According to the manufacturer's protocols, the normal upper limit was $1.5 \mu \mathrm{g} / 1$ for SCC-Ag and $3.3 \mathrm{ng} / \mathrm{ml}$ for CYFRA 21-1 (16-18). Patients were divided into low and high subsets according to the normal upper limit of the prognostic factors. Patients' prognostic factors were obtained prior to treatment (surgery or chemotherapy).

Pathological analysis. According to the International Union Against Cancer (UICC) tumor-lymph node-metastasis (TNM) classification for esophageal cancer (23), 29 patients were graded with stage I, 22 with stage II, and 33 with stage III cancer. An R0 resection, defined as complete tumor excision, was performed for all patients.

Statistical analysis. The Mann-Whitney U test, Chi-squared test and Fisher's exact probability test were used to compare the clinicopathological characteristics of the two groups. Spearman's rank correlation coefficient was used to assess the correlation between the two numerical parameters. A strong correlation between the parameters and occurrence of postoperative complications was estimated using the logistic-regression analysis. Long-term survival was calculated using the Kaplan-Meier method, and the prognostic difference between the two groups was compared using the log-rank test. Multivariate Cox regression analysis was used for all parameters that were found to be significant using univariate analysis. $\mathrm{P}<0.05$ was considered to indicate a statistically significant difference.

\section{Results}

The clinical characteristics of the 84 patients are shown in Table I. Surgical complications were detected in 36 patients (morbidity was 42.9\%). Two patients died due to surgical complications, and the mortality was $2.4 \%$. Anastomotic leakage was 
Table I. Clinical characteristics of 84 patients with thoracic esophageal squamous cell carcinomas who underwent esophagectomy and three-field lymph node dissection.

\begin{tabular}{lc}
\hline Characteristic & Clinical data \\
\hline Age (mean; median, range, years) & $65.7(66,49-78)$ \\
Gender (male/female) & $73 / 11$ \\
Operation time (mean; median, range, minutes) & $649(625,420-984)$ \\
Intraoperative blood loss (mean; median, range, ml) & $475.3(320,10-3,020)$ \\
Number of dissected lymph nodes (Mean, median, range) & $50.6(49,14-110)$ \\
Histological tumor stage (I/II/III) & $29 / 22 / 33$ \\
Postoperative complications (yes/no) & $36 / 48$ \\
Postoperative hospital stay (mean; median, range, days) & $50.3(31,10-478)$
\end{tabular}

Table II. Multivariate analysis of predictors of postoperative complications.

\begin{tabular}{lccr}
\hline & P-value & Odds ratio & 95\% confidence interval \\
\hline Advanced age & 0.608 & 0.741 & $0.236-2.326$ \\
Male & 0.438 & 0.520 & $0.100-2.712$ \\
Neoadjuvant chemotherapy & 0.383 & 1.848 & $0.466-7.299$ \\
Open thoracotomy & 0.083 & 5.155 & $0.806-33.333$ \\
Long operation time & 0.166 & 2.421 & $0.693-8.475$ \\
Large intraoperative blood loss & 0.705 & 0.574 & $0.175-3.257$ \\
Large number of dissected lymph nodes & 0.168 & 0.842 & $0.108-1.083$ \\
Tumor stage III & 0.127 & 0.395 & $0.120-1.301$ \\
High GPS & 0.254 & 3.300 & $0.425-25.641$ \\
High NLR & 0.533 & 0.593 & $0.115-3.058$ \\
Low PNI & 0.681 & 0.767 & $0.217-2.710$ \\
High SCC-Ag & 0.103 & 0.324 & $0.084-1.253$ \\
High CYFRA 21-1 & 0.007 & 9.346 & $1.825-47.619$
\end{tabular}

GPS, Glasgow Prognostic Score; NLR, neutrophil/lymphocyte ratio; PNI, prognostic nutritional index; SCC-Ag, squamous cell carcinoma antigen; CYFRA 21-1, cytokeratin 19 fragments.

detected in 25 patients, pneumonia in four, chylothorax in four, and necrosis of the substituted esophagus was observed in three.

A total of 77 patients were GPS $=0$, six were GPS $=1$ and one was GPS $=2$. Therefore, high GPS was detected in seven of the patients and the remaining 77 were characterized as low GPS. The mean NLR was 2.3 (median 2.3, range: 0.6-5.8). Twenty-six patients were classified in the high NLR group (NLR $\geq 3$ ), and 58 were in the low NLR group. The mean PNI was 51.2 (median 50.6, range: 36.4-72.2). Fifty patients were classified in the high PNI group (PNI $\geq 50$ ), and 34 were in the low PNI group. The mean SCC-Ag was $1.6 \mu \mathrm{g} / 1$ (median 1.2, range: 0.3-9.1). Thirty-two patients were classified in the high SCC-Ag group (SCC-Ag $\geq 1.5 \mu \mathrm{g} / \mathrm{l}$ ), and 52 were in the low SCC-Ag group. The mean CYFRA 21-1 was $2.3 \mathrm{ng} / \mathrm{ml}$ (median 1.2, range: 0.3-9.1). Fifteen patients were classified in the high CYFRA 21-1 group (CYFRA 21-1 $\geq 3.3 \mathrm{ng} / \mathrm{ml}$ ) and 69 in the low CYFRA 21-1 group. Thirteen factors that were thought to correlate with the occurrence of postoperative complications were analyzed using the logistic regression test (Table II). The median values of patient age, operation time, intraoperative blood loss and number of dissected lymph nodes were used as cut-offs. Strong correlations between the occurrence of postoperative complications and open thoracotomy $(\mathrm{P}=0.083)$ and high-serum CYFRA 21-1 $(\mathrm{P}=0.007)$ were observed. In 15 patients with high-serum CYFRA 21-1, postoperative complications were detected in $11(73.3 \%)$; on the other hand, in 69 patients with low-serum CYFRA 21-1, postsurgical complications were detected in 25 (36.2\%). A positive correlation was detected between SCC-Ag and CYFRA 21-1 $(\mathrm{P}=0.359, \mathrm{P}=0.001)$, and a strong negative correlation was detected between NLR and PNI $(\mathrm{P}=-0.449$, $\mathrm{P}<0.001)$.

The 5-year disease-free survival (DFS) rate and 5-year overall survival (OS) rate of all patients were 52.2 and $50.8 \%$, respectively. The long-term outcomes were compared between the two groups (Table III). Postoperative complications did not affect DFS or OS. Furthermore, the SCC-Ag and CYFRA 21-1 values did not correlate with survival. NLR and PNI were recognized as strong prognostic factors from the univariate analysis. Table IV shows the multivariate survival analysis of the factors that were detected as significant in DFS using univariate analysis 
Table III. The 5-year disease-free survival rate and 5-year overall survival rate of the two groups.

\begin{tabular}{|c|c|c|c|c|c|}
\hline Parameters & No. & 5-year DFS (\%) & P-value & 5-year OS (\%) & P-value \\
\hline \multicolumn{6}{|l|}{ Age } \\
\hline High & 45 & 56.2 & 0.486 & 46.3 & 0.478 \\
\hline Low & 39 & 48 & & 55.1 & \\
\hline \multicolumn{6}{|l|}{ Gender } \\
\hline Male & 73 & 50.3 & 0.365 & 48.1 & 0.248 \\
\hline Female & 11 & 63.6 & & 69.3 & \\
\hline \multicolumn{6}{|l|}{ Tumor stage } \\
\hline I and II & 51 & 73.8 & $<0.001$ & 67.9 & $<0.001$ \\
\hline III & 33 & 19.6 & & 19.5 & \\
\hline \multicolumn{6}{|c|}{ Postoperative complication } \\
\hline Yes & 36 & 49.4 & 0.833 & 44.4 & 0.13 \\
\hline No & 48 & 53.6 & & 55.5 & \\
\hline \multicolumn{6}{|l|}{ GPS } \\
\hline High & 7 & 85.7 & 0.123 & 85.7 & 0.238 \\
\hline Low & 77 & 49 & & 49.0 & \\
\hline \multicolumn{6}{|l|}{ NLR } \\
\hline High & 26 & 28.8 & $<0.001$ & 28.1 & $<0.001$ \\
\hline Low & 58 & 62.8 & & 60.2 & \\
\hline \multicolumn{6}{|l|}{ PNI } \\
\hline High & 50 & 60.3 & 0.026 & 53.6 & 0.017 \\
\hline Low & 34 & 39.5 & & 43.0 & \\
\hline \multicolumn{6}{|l|}{ SCC-Ag } \\
\hline High & 32 & 50.9 & 0.985 & 50.8 & 0.871 \\
\hline Low & 52 & 52.9 & & 50.7 & \\
\hline \multicolumn{6}{|c|}{ CYFRA 21-1 } \\
\hline High & 15 & 40 & 0.271 & 42.8 & 0.956 \\
\hline Low & 69 & 55.1 & & 51.3 & \\
\hline
\end{tabular}

DFS, disease-free survival; OS, overall survival; GPS, Glasgow Prognostic Score; NLR, neutrophil/lymphocyte ratio; PNI, prognostic nutritional index; SCC-Ag, squamous cell carcinoma antigen; CYFRA 21-1, cytokeratin 19 fragments.

Table IV. Multivariate disease-free survival analysis of patients.

\begin{tabular}{lcrr}
\hline Parameter & P-value & Odds ratio & 95\% confidence interval \\
\hline Tumor stage III & $<0.001$ & 5.714 & $2.793-11.628$ \\
High NLR & 0.03 & 2.426 & $1.091-5.394$ \\
Low PNI & 0.369 & 1.431 & $0.655-3.125$ \\
\hline
\end{tabular}

NLR, neutrophil/lymphocyte ratio; PNI, prognostic nutritional index.

(Table III). Among the prognostic factors, high NLR was identified as a poor prognostic factor independent of the tumor stage.

\section{Discussion}

Esophagectomy plus three-field lymph node dissection has been the standard surgical regimen for the treatment of thoracic ESCCs. However, high morbidity or mortality and poor prognosis of patients should be considered prior to opting for surgical treatment. In the present study, postoperative morbidity and mortality were 42.9 and $2.4 \%$, respectively. In these cases of high morbidity, it was determined that the preoperative high-serum CYFRA 21-1 was a clear indicator of the occurrence of postoperative complications. CYFRA 21-1 is 
a cytoskeleton marker with a low-molecular weight (40 kDa), and is one of the serum tumor markers of squamous cell carcinomas (16-18). Recently, Vercauteren et al (24) reported that increased levels of serum CYFRA 21-1 were correlated with pulmonary fibrosis. Thus, a high-serum CYFRA 21-1 level may correlate with tissue disorder at the anastomotic site, such as non-development of micro-vascularization. A detailed study is expected to be performed regarding the biological significance of serum CYFRA 21-1. In patients with high-serum CYFRA 21-1, supercharged microvascular anastomoses between the cervical neck and the substitute esophagus should be considered to avoid the occurrence of anastomotic leakage.

Tumor stage III, high NLR and low PNI were identified to be poor DFS and OS factors in patients with operable thoracic ESCCs. These findings have been reported previously (25-27). Specifically, it our study has revealed, via the multivariable analysis of our present cohort, that NLR was an important prognostic marker in patients with thoracic ESCCs independent of the tumor stage. Han et al (28) identified that high pretreatment NLRs were significantly associated with high levels of neutrophil infiltration and low $\mathrm{CD}^{+}{ }^{+}$T-cell infiltration into glioblastomas, and a strong correlation with shorter survival times in patients with glioblastoma. Neutrophil infiltration into various tumors serves an important role in stimulating tumor growth, angiogenesis and metastasis $(29,30)$. In addition, neutrophils may suppress immune function by inhibiting the cytotoxic activity of $\mathrm{CD}^{+}$ T-cells and natural killer cells by enhancing the suppressive activities of $\mathrm{CD}^{+}$suppressor $\mathrm{T}$ cells in the infiltrating tissues. These findings may indicate that the pretreatment NLR measured in peripheral blood samples from patients will be a useful marker of local host immunity.

This study had a number of limitations. First, the number of patients with resectable thoracic ESCCs in our series was small. Secondly, our study was a retrospective analysis, and there is a possibility that the results from our study may have bias. However, our results do indicate that, in patients with preoperative high-serum CYFRA 21-1, more attention will need to be paid to the occurrence of postoperative complications. Therefore, in such cases, anastomosis between blood vessels of the substitute esophagus and cervical vessels should be recommended. Additionally, our results may indicate that, in patients with high preoperative NLR, effective adjuvant chemoradiotherapy should be considered to prolong the patients' survival, even of stage I or II patients.

\section{References}

1. Ando N, Ozawa S, Kitagawa Y, Shinozawa Y and Kitajima M Improvement in the results of surgical treatment of advanced squamous esophageal carcinoma during 15 consecutive years. Ann Surg 232: 225-232, 2000.

2. Tachibana M, Kinugasa S, Yoshimura H, Shibakita $M$, Tonomoto Y, Dhar DK and Nagasue N: Clinical outocomes of extended esophagectomy with three-field lymph node dissection for esophageal squamous cell carcinoma. Am J Surg 189: 98-109, 2005

3. Shimada H, Okazumi S, Matsubara H, Nabeya Y, Shiratori T, Shimizu T, Shuto K, Hayashi H and Ochiai T: Impact of the number and extent of positive lymph nodes in 200 patients with thoracic esophageal squamous cell carcinoma after three-field lymph node dissection. World J Surg 30: $1441-1449,2006$
4. Higuchi K, Koizumi W, Tanabe S, Sasaki T, Katada C, Azuma M, Nakatani K, Ishido K, Naruke A and Ryu T: Current management of esophageal squamous-cell carcinoma in Japan and other countries. Gastrointest Cancer Res 3: 153-161, 2009.

5. Grimm JC, Valero V III and Molena D: Surgical indications and optimization of patients for resectable esophageal malignancies. J Thorac Dis 6: 249-257, 2014.

6. Reed CE: Surgical management of esophageal carcinoma. The Oncologist 4: 96-105, 1999.

7. Hwang JE, Kim HN, Kim DE, Choi HJ, Jung SH, Shim HJ, Bae WK, Hwang EC, Cho SH and Chung IJ: Prognostic significance of a systemic inflammatory response in patients receiving first-line palliative chemotherapy for recurred or metastatic gastric cancer. BMC Cancer 11: 489, 2011.

8. Urba S, Gatz J, Shen W, Hossain A, Winfree K, Koustenis A, Peterson P and Cohen EE: Quality of life scores as prognostic factors of overall survival in advanced head and neck cancer: Analysis of a phase III randomized trial of pemetrexed plus cisplatin versus cisplatin monotherapy. Oral Oncol 48: 723-729, 2012.

9. McMillan DC: The systemic inflammation-based Glasgow Prognostic Score: A decade of experience in patients with cancer. Cancer Treat Rev 39: 534-540, 2013.

10. Kishiki T, Masaki T, Matsuoka H, Kobayashi T, Suzuki Y, Abe N, Mori T and Sugiyama M: Modified Glasgow prognostic score in patients with incurable stage IV colorectal cancer. Am J Surg 206: 234-240, 2013.

11. Chua W, Charles KA, Baracos VE and Clarke SJ: Neutrophil/lymphocyte ratio predicts chemotherapy outcomes in patients with advanced colorectal cancer. Br J Cancer 104: 1288-1295, 2011.

12. Yamanaka T, Matsumoto S, Teramukai S, Ishiwata R, Nagai Y and Fukushima M: The baseline ratio of neutrophils to lymphocytes is associated with patient prognosis in advanced gastric cancer. Oncology 73: 215-220, 2007.

13. Ubukata H, Motohashi G, Tabuchi T, Nagata H, Konishi S and Tabuchi T: Evaluation of interferon- $\gamma /$ interleukin- 4 ratio and neutrophil/lymphocyte ratio as prognostic indicators in gastric cancer patients. J Surg Oncol 102: 742-747, 2010.

14. Nozoe T, Kohno M, Iguchi T, Mori E, Maeda T, Matsukuma A and Ezaki T: The prognostic nutritional index can be a prognostic indicator in colorectal carcinoma. Surg Today 42: 532-535, 2012.

15. Ikeguchi M, Urushibara S, Shimoda R, Yamamoto M, Maeta Y and Ashida K: Inflammation-based prognostic scores and nutritional prognostic index in patients with locally-advanced unresectable colorectal cancer. World J Surg Oncol 12: 210, 2014.

16. Tsuchita Y, Onda M, Miyashita M and Sasajima K: Serum level of cytokeratin 19 fragment (CYFRA 21-1) indicates tumour stage and prognosis of squamous cell carcinoma of the oesophagus. Med Oncol 16: 31-37, 1999.

17. Nabeya Y, Shimada H, Okazumi S, Matsubara H, Gunji Y, Suzuki T and Ochiai T: Serum cross-linked carboxyterminal telopeptide of type I collagen (ICTP) as a prognostic tumor marker in patients with esophageal squamous cell carcinoma. Cancer 94: 940-949, 2002.

18. Cao X, Zhang L, Feng GR, Yang J, Wang RY, Li J, Zheng XM and Han YJ: Preoperative Cyfra21-1 and SCC-Ag serum titers predict survival in patients with stage II esophageal squamous cell carcinoma. J Transl Med 10: 197, 2012.

19. Ando N, Iizuka T, Ide H, Ishida K, Shinoda M, Nishimaki T, Takiyama W, Watanabe H, Isono K, Aoyama N, et al: Surgery plus chemotherapy compared with surgery alone for localized squamous cell carcinoma of the thoracic esophagus: A Japan Clinical Oncology Group Study-JCOG9204. J Clin Oncol 21: 4592-4596, 2003.

20. Fujiwara Y, Yoshikawa R, Kamikonya N, Nakayama T, Kitani K, Tsujie M, Yukawa M, Inoue M and Yamamura T: Trimodality therapy of esophagectomy plus neoadjuvant chemoradiotherapy improves the survival of clinical stage II/III esophageal squamous cell carcinoma patients. Oncol Rep 28: 446-452, 2012.

21. Duan H, Zhang X, Wang FX, Cai MY, Ma GW, Yang H, Fu JH, Tan ZH, Meng YQ, Fu XY, et al: Prognostic role of neutrophil-lymphocyte ratio in operable esophageal squamous cell carcinoma. World J Gastroenterol 21: 5591-5597, 2015.

22. Sun P, Zhang F, Chen C, An X, Li YH, Wang FH and Zhu ZH: Comparison of the prognostic values of various nutritional parameters in patients with esophageal squamous cell carcinoma from Southern China. J Thorac Dis 5: 484-491, 2013.

23. Sobin LH and Wittekind CH: UICC TNM classification of malignant tumours. 6th edn. Hoboken, New Jersey: John Wiley \& Sons; 2002. 
24. Vercauteren IM, Verleden SE, McDonough JE, Vandermeulen E, Ruttens D, Lammertyn EJ, Bellon H, De Dycker E, Doomes C, Yserbyt J, et al: CYFRA 21.1 in bronchoalveolar lavage of idiopathic pulmonary fibrosis patients. Exp Lung Res 41: 459-465, 2015.

25. Hirahara N, Matsubara T, Hayashi H, Takai K, Fujii Y and Tajima Y: Impact of inflammation-based prognostic score on survival after curative thoracoscopic esophagectomy for esophageal cancer. Eur J Surg Oncol 41: 1308-1315, 2015.

26. Yodying $\mathrm{H}$, Matsuda A, Miyashita M, Matsumoto S, Sakurazawa N, Yamada M and Uchida E: Prognostic significance of neutrophil-to-lymphocyte ratio and platelet-to-lymphocyte ratio in oncologic outocomes of esophageal cancer: A systematic review and meta-analysis. Ann Surg Oncol 23: 646-654, 2016

27. Liu JS, Huang Y, Yang X and Feng JF: A nomogram to predict prognostic values of various inflammatory biomarkers in patients with esophageal squamous cell carcinoma. Am J Cancer Res 5: 2180-2189, 2015.
28. Han S, Liu Y, Li Q, Li Z, Hou H and Wu A: Pre-treatment neutrophil-to-lymphocyte ratio is associated with neutrophil and T-cell infiltration and predicts clinical outcome in patients with glioblastoma. BMC Cancer 15: 617, 2015.

29. Bekes EM, Schweighofer B, Kupriyanova TA, Zajac E, Ardi VC, Quigley JP and Deryugina EI: Tumor-recruited neutrophils and neutrophil TIMP-free MMP-9 regulate coordinately the levels of tumor angiogenesis and efficiency of malignant cell intravasation. Am J Pathol 179: 1455-1470, 2011.

30. Dumitru CA, Lang S and Brandau S: Modulation of neutrophil granulocytes in the tumor microenvironment: Mechanisms and consequences for tumor progression. Semin Cancer Biol 23: 141-148, 2013. 